\title{
The Intra-Myocardial Distance Function Used in Inverse Computations of the Timing of Depolarization and Repolarization
}

\author{
A van Oosterom ${ }^{1}, \mathrm{P}$ van Dam $^{2}$ \\ ${ }^{1}$ Dept of Cardiology, University of Lausanne, Lausanne, VD, Switzerland \\ ${ }^{2}$ Vitatron Research, Arnhem, The Netherlands
}

\begin{abstract}
This paper introduces novel methods for finding an initial solution required for the inverse computation of the timing of the depolarization and repolarization of the atria and the ventricles, as well as for constraining the subsequent iterative solution. Both methods make use of the shortest path between any two points on the heart's surface while traveling through the myocardium.
\end{abstract}

\section{Introduction}

The inverse computation of the timing of depolarization of the ventricular myocytes on the basis of observed body surface potentials has been shown to be feasible[1,2]. More recently, this method has been extended to the inverse computation of the timing of ventricular repolarization based on the equivalent double layer surface source model (EDL)[3], as well as to atrial activation[4].

The inverse computation of timing constitutes a nonlinear parameter estimation problem, and as such requires an initial estimate. The subsequent iterative solution requires the inclusion of a constraint. This paper introduces novel methods for the handling of both requirements, both based on the intra-myocardial distance function: the shortest distance between any point on the myocardial surface and all other points on the same surface while passing through the myocardium. The effectiveness of the methods is demonstrated in their application to the inverse computation of the timing of depolarization and repolarization of the atria of a healthy subject.

\section{Methods}

\section{Forward formulation}

The inverse computation of cardiac electric sources requires an expression describing the relationship between the assumed source nature and the resulting potentials at the observation points of interest (the field points). In the current application, the field points are situated on the torso boundary.

The source description used is the instantaneous distribution of transmembrane potentials (TMPs) of the myocytes at the heart surface $S_{\mathrm{h}}$, which is taken to be the surface bounding the myocardium, i.e., the closed surface bounding epicardium and endocardium. The time course of the TMP at a specific location, $x$, on this surface is specified by an analytical expression based on the product of a number of logistic functions[5]. The parameters of this expression have been tuned to wave forms observed in electrophysiological studies. Separate versions were derived for modeling the transmembrane potentials of ventricular and atrial myoctes in such a manner that the wave forms of individual myocytes could be specified by two parameters only: the depolarization time $\delta$ and the repolarization time $\rho$. These correspond to the timing of the maximum up slope and the maximum down slope during phase 3 of the TMP, respectively. The source strength of any of the source elements may, accordingly, be expressed by

$$
S(x, t)=S(t ; \delta(x), \rho(x))
$$

The effect of these sources as potentials $\Phi(y, x)$ observed at arbitrary field points, $y$, is governed by the passive volume conduction effects of the tissues surrounding the heart. These can be expressed by a transfer function $A(y, x)$, which permits the genesis of the potentials $\Phi(y, t)$, the forward formulation, to be written as

$$
\Phi(y, t)=\int_{S_{\mathrm{h}}} A(y, x) S(t ; \delta(x), \rho(x)) \mathrm{d} S_{\mathrm{h}} .
$$

As in related earlier work[1-3;6], the numerical implementation of Eqn.2 was carried out by using a discretized version of the surface $S_{\mathrm{h}}$, a triangulated representation with its nodes $j, j=1 \ldots n$, carrying the local source strengths as specified by Eqn.1.

The transfer function was computed by means of the boundary element method. This method was applied to an 
inhomogeneous model of the conductivity inside the thorax, which accounted for the higher and lower electric conductivity of the blood inside the cavities and lung tissue, respectively. For discrete observation points Eqn.2 can be represented by the matrix multiplication

$$
\Phi=\mathbf{A} \mathbf{S}
$$

in which matrix element $S_{j t}=S\left(t ; \delta_{j}, \rho_{j}\right)$ represents the source strength at node $j$ at discrete time instant $t$.

\section{Inverse formulation}

Equation 3 provides the entry to computing the timing of depolarization and repolarization from observed body surface potentials. By denoting the potentials observed by means of any lead system by the matrix $\mathbf{V}$, the solution is taken to be the set $\left(\delta_{j}, \rho_{j}\right)$ that minimizes the sum of the squared differences between all elements of $\mathbf{V}$ and those of $\mathbf{A S}(\boldsymbol{\delta}, \boldsymbol{\rho})$ [1-3], i.e.,

$$
(\boldsymbol{\delta}, \boldsymbol{\rho})=\underset{\boldsymbol{\delta}, \boldsymbol{\rho}}{\arg \min }\|\mathrm{V}-\mathrm{A} \mathrm{S}(\boldsymbol{\delta}, \boldsymbol{\rho})\|_{\mathrm{F}}^{2},
$$

with $\mathrm{F}$ denoting the Frobenius norm of a matrix. This can be classed as a parameter estimation problem.

The problem in hand involves two complexities. The first one is as follows. Since a change in any of the $\delta_{j}, \rho_{j}$ values results in a shifting and/or stretching of the source strength at node $j$, the potentials $\boldsymbol{\Phi}$ depend non-linearly on the parameter vectors $[\boldsymbol{\delta} \boldsymbol{\rho}]$. This means that we are dealing with a non-linear parameter estimation problem. Hence, iterative solution methods need to be used, requiring an initial estimate.

The second complexity relates to the nature of the transfer matrix A, which is generally ill-conditioned, as well as to the fact that generally the number of electrodes used for sampling the potential distribution is smaller than the number of parameters that one may wish to estimate. Both aspects demand the inclusion of a constraint on the nature of the solution, aimed at guarding its realism. This can be effected by including a penalty function in Eqn.4:

$$
\begin{aligned}
& (\boldsymbol{\delta}, \boldsymbol{\rho})=\underset{\boldsymbol{\delta}, \boldsymbol{\rho}}{\arg \min }\left(\|\mathrm{V}-\mathrm{A} S(\boldsymbol{\delta}, \boldsymbol{\rho})\|_{\mathrm{F}}^{2}+\right. \\
& \left.\lambda^{2}\|\mathrm{C}(\boldsymbol{\delta}, \boldsymbol{\rho})\|_{\mathrm{F}}^{2}\right),
\end{aligned}
$$

with $\mathbf{C}$ specifying the type of constraint and $\lambda$ its weight.

Below, the intra-myocardial distance function is introduced for handling both complexities.

\section{The intra-myocardial distance}

The intra-myocardial distance used was taken to be the shortest distance between any two nodes of the triangular mesh representing $S_{\mathrm{h}}$ while passing along a route lying entirely within the myocardium. The mesh of the triangulated surface constitutes a connected graph, for which the distances between any two nodes can be found from its adjacency matrix $\mathbf{M}$, by using the shortest path algorithm $[7,8]$. For any pair of nodes $(i, j)$, the value of the corresponding element of $\mathbf{M}, M_{i j}$, was set to be their distance in 3-D space, but only if the straight line connecting the pair did not intersect any of the triangles not carrying nodes $i$ or $j$; else $M_{i j}$ was set to zero. This procedure produces the symmetric matrix D, whose elements $D_{i j}$ constitute the lengths of all shortest paths.

The complexity of the geometry of the heart surface requires its numerical representation be based on numerous nodes. For $n$ nodes, the adjacency matrix contains $n(n+1) / 2$ individual entries, all of which need to be scrutinized for possible intersection with any of the (approximately) $2 n$ triangles. A dedicated program was developed and tested using Matlab $\left(\right.$ MATLAB $\left.^{\circledR}\right)$, and streamlined in a $\mathrm{C}$ code. This code may also be called while using Matlab. (All codes are available on request). For $n=1,500$ the setting up of the distance matrix took 3 hours and 40 minutes when running on a PC running at $2.2 \mathrm{GHz}$ with a $1 \mathrm{~Gb}$ internal memory.

\section{Deriving the initial estimate of $\delta$ from the intra- myocardial distance}

The geometry of the boundaries of the myocardial tissue in a healthy normal human male (age 22, height $196 \mathrm{~cm}$, weight $82 \mathrm{~kg}$ ), was recorded by means of Magnetic Resonance Imaging (MRI). The discretization of the boundaries of the myocardium resulted in a triangulated version of $S_{\mathrm{h}}$. Any column $j$ of the matrix D describes the distances between node $j$ and all other nodes. By assuming node $j$ to be the focus of an activation process propagating at uniform velocity $v$, the timing of depolarization at all other nodes was taken to be

$$
\boldsymbol{\delta}_{j}, \text { with } \delta_{i j}=d_{i j} / v \text {. }
$$

By taking all nodes, one at a time, as the focus, a collection of $n$ different activation patterns is defined. Each of these was used, again one at a time, to simulate potentials on lead positions on the thorax, based on $\boldsymbol{\Phi}=$ A $\mathbf{S}\left(\boldsymbol{\delta}_{\mathrm{j}}\right)$, as in Eqn.3. Since the objective was to find an initial estimate for the timing of depolarization only, the analytical function specifying $S(t)$ was restricted to modeling the upstroke of the TMP, which reduced the required computation time.

The transfer matrix was computed from a multicompartmental representation of the thorax of the subject studied, which included the lower conductivity of the lungs as well as the higher conductivity of the ventricular 


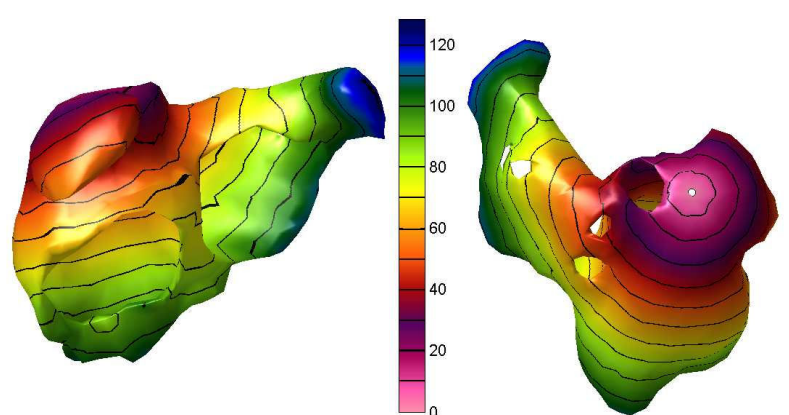

Figure 1. Initial estimate of the timing of atrial depolarization based on the intra-mural distances. Isochrones drawn at $10 \mathrm{~ms}$ intervals. White circle indicates the node identified as the focus. Left panel: anterior view; right panel: posterior view.

and atrial cavities.

The initial estimate was taken to be the pattern that resulted in the highest (positive) linear correlation between the simulated potentials and the ones measured on the subject by means of a 64 lead recording system[9]. The correlation was computed by including all individual potential values of the 64 leads at all NT samples at $1 \mathrm{~ms}$ distance in the time interval from the onset of depolarization, to the $\mathrm{J}$ point, treated as a vector of dimension 64NT. All instantaneous lead potentials, the measured as well as the simulated ones, were referred to a zero mean reference.

\section{The regularization operator based on the intra- myocardial distance}

Previous studies on the inverse computation of the timing of depolarization and repolarization employed the Surface Laplacian operator for regularizing the solution[1-3]. This operator penalizes large differences between the value of the timing at any node and the mean of the values at the nodes surrounding it on the surface $S_{\mathrm{v}}$. When applied to computing the timing of ventricular electrical activity this operator was found to be very effective. However, when applied to the timing of atrial electrical activity, matters were entirely different. The reason was found to lie in the much smaller wall thickness of the atria $(\sim 2 \mathrm{~mm})$ as compared to that of the ventricles $(\sim 1.5 \mathrm{~cm})$, with the activation wave front propagating mainly parallel to the wall, and transmural differences in timing being small. Since nodes that are widely spaced when measured over the atrial surface, e.g., from endocardium to epicardium, can be close in 3D space (transmurally), a surface based constraint operator fails. It is here that the intramural distance function proved to be more appropriate.

The new regularization operator was based on the reciprocals of the squared values of distances in 3-D space: the elements of the distance matrix D. By writing $w_{i j}=1 / d^{2}{ }_{i j}$ for $i \neq j$ and $w_{i i}=0$, the elements of the constraint matrix $\mathbf{C}$ were taken to be

$$
c_{i j}=w_{i j} / \sum_{j=1}^{n} w_{i j} \quad \text { for } i \neq j, \quad \text { and } c_{i i}=-1 \text {. }
$$

\section{Results}

\section{Application to the atria}

The application of the new method for finding the initial estimate of the timing of depolarization of the atria led to the solution shown in Figure 1. The geometry is represented by 1,504 nodes, the vertices of 3,028 triangles, and has the topology of a sixth-order doughnut. It includes the main anatomical details where no electrical activity is present: the tricuspid and mitral valves and the entries of inferior vena cava, inferior vena cava, and the 4 pulmonary veins.

For this geometry, the longest distance between any pair of nodes is $13.9 \mathrm{~cm}$. The total surface area (endo+epi) is $341 \mathrm{~cm}^{2}$; the total volume of the myocardial tissue is $46.2 \mathrm{~cm}^{3}$, which yields a crude estimate of the wall thickness of $46.2 /(0.5 \times 341)=0.27 \mathrm{~cm}$.

The node identified as the focus was found close to the right hand side of the entry of the vena cava superior, the area where the sinus node usually resides. The longest intra-myocardial distance between this focus and all other nodes is $9.65 \mathrm{~cm}$. The timing of the $J_{a}$ point as identified from the $\operatorname{RMS}(t)$ curve based on all recorded leads was taken as the timing of the latest atrial regions activated. This occurred at $120 \mathrm{~ms}$ after onset of the $\mathrm{P}$ wave as identified in the $\operatorname{RMS}(t)$ curve. This corresponds to a mean propagation velocity of $0.8 \mathrm{~m} / \mathrm{s}$; the value that was subsequently used in Eqn.6

For this optimal focus, the simulated potentials in the 64 lead system showed a correlation coefficient of $\mathrm{R}=0.88$ with the measured ones. The rms value of the difference between simulated and measured potentials relative to that of the measured ones, $\mathrm{RD}$, was 0.91 . This high a value would have previously led us to reject the initial estimate. However, subsequent iterations of the inverse procedure led, in a stable manner, to $\mathrm{RD}=0.213$., with $\mathrm{R}=0.977$. The number of iterations required for reaching convergence (relative changes in subsequent solutions falling below $10^{-4}$ ) was 25 , requiring 7 minutes of computing time. The isochrones of this solution are shown in Figure 2. The qualitative nature of this solution is in agreement with published literature data[10]. The statistics of differences (in $\mathrm{ms}$ ) between the initial estimate (Figure 1) and the final on (Figure 2) were: mean \pm SD $0.1 \pm 1.8$; range $\left[\begin{array}{ll}-8.7 & 19\end{array}\right]$, with the larger deviations showing up around the orifices of the mitral and tricuspid valves. These results were obtained by using the constraint operator as defined in Eqn.7.

The solution proved to be insensitive to the value of 


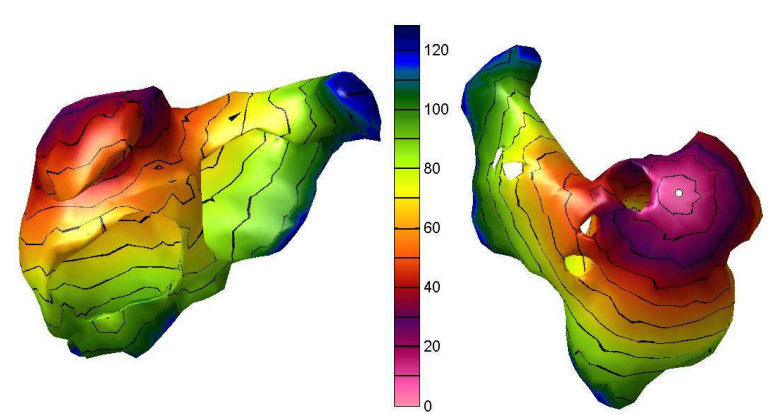

Figure 2. The timing of atrial depolarization as found after 23 iterations. Remaining legend as in Figure 2.

the regularization parameter $\lambda$. Even when using $\lambda=0$, the un-regularized situation, the solution, while appearing more 'patchy', had the same global appearance.

The involved minimization was carried out by means of a dedicated minimization program based on the Marquardt algorithm[11].

\section{Application to the ventricles}

The method for finding an initial estimate was also applied to the inverse computation of the timing of ventricular depolarization. The exhaustive search for the focus, here based on $v=1.15$, yielded a maximum correlation between measured and simulated signals identified a node on the septum of left ventricle, of $\mathrm{R}=$ 0.826 , at a location on the left ventricular aspect of the septal wall, consistent with known electrophysiology.

The subsequently, the iteration procedure was applied alternately to optimising the depolarisation and repolarization times at the nodes, while using the Surface Laplacian constraint. Convergence was reached at an RD value of 0.115 , corresponding to a correlation coefficient of 0.993 . These values are superior to the values found previously[1-3]. These previous procedures, based on different initial estimates, failed to localize the earliest sites of left septal ventricular activation. Moreover, the new initial estimate produced stable convergence to the final solution without requiring elaborate tuning of the regularization parameter.

\section{Discussion and conclusions}

The intra-myocardial distance function is a generalization of a previous version that involved distances over the atrial surface only[7]. The additional computational burden of including the thickness of the atrial wall increased the computational burden $\left(\sim n^{3}\right)$, but this was compensated by the reduction in the subsequent computational demand on the inverse procedure, and the increased quality of the results. Both novel elements in the inverse computation of the timing of depolarization proved to be highly effective. The method deriving the initial estimate based on the intra-myocardial distance may be viewed as a forward (inside-outside) approach based on the main electrophysiological property of propagated excitation. This is in contrast to the previously used methods, which are essentially inverse based approaches, based exclusively on the theory of data processing, without clear elements based on electrophysiology. Similarly, the adaptation of the novel regularization operator was inspired by the demands arising from electrophysiology.

\section{References}

[1] Huiskamp GJM, van Oosterom A. The Depolarization Sequence of the human heart surface computed from measured body surface potentials. IEEE Trans Biomed Eng 1988;BME_35:1047-58.

[2] Huiskamp GJM, Greensite F. A New method for Myocardial Activation Imaging. IEEE Trans Biomed Eng 1997;BME_44:433-46.

[3] van Oosterom, A. Genesis of the T wave as based on an equivalent surface source model. $\mathrm{J}$ Electrocardiol 2001;34S:217-27.

[4] Modre R, Tilg B,Fisher G, Hanser F,Messnarz B, Seger M, Schocke MFH, Berger T, Hintringer F, Roithinger F: Atrial Noninvasive Activation Mapping of Paced Rhythm Data. J Cardiovasc Electrophysiol 2003;14:712-719.

[5] van Oosterom A, Jacquemet V. A parameterized description of transmembrane potentials used in forward and inverse procedures. Folia Cardiol 2005;12-S_D:O358. (CD-rom, also available from: www.lausanneheart.ch)

[6] van Oosterom A., Jacquemet V. Genesis of the P wave: Atrial Signals as Generated by the Equivalent Double Layer Source Model. Europace 2005;7:S83-92.

[7] van Dam P. van Oosterom A. Atrial excitation assuming uniform propagation. $\mathrm{J}$ Cardiovasc Electrophysiol 2003;14:S166-71.

[8] Wilson RJ. Introduction to Graph Theory. 3rd edition. Longman Scientific \& Technical, Harlow, Essex, 1985.

[9] Heringa A, Uijen GJH, van Dam RT. A 64-channel system for body surface potential mapping. In: Z Antalózcy and I Préda (edts), Electrocardiology 1981:297-301, Academia Kiado, Budapest, 1982.

[10] Ponti Rd, Ho SY, Salerno-Uriarte JA, Tritto M, Spadacini G: Electroanatomic analysis of sinus impulse propagation in normal human atria. Journal of Cardiovascular Electrophysiology. 2002;13:1-10.

[11] Marquardt DW. An algorithm for least-squares estimation of non-linear parameters. J Soc Indust Appl Math 1963;2:431-41.

\author{
Address for correspondence \\ A. van Oosterom \\ E-mail: adriaan.vanoosterom@epfl.ch \\ EPFL-STI-ITS-LTS1 \\ ELD 241 (Bâtiment ELD) \\ CH 1015 Lausanne
}

